\title{
Comparison of Nutric Score, Nutritional Risk Screening (NRS) 2002 and Subjective Global Assessment (SGA) in the ICU: a Cohort Study
}

\author{
Sanjith Saseedharan* \\ University: s I raheja hospital, Address: dept of critical care, s I raheja hospital, raheja rugnalaya marg mahim, mumbai- 400016, India
}

Received: 01 October, 2019; Accepted: 03 December, 2019; Published: 10 December, 2019

*Corresponding author: Sanjith Saseedharan, University: s l raheja hospital, Address: dept of critical care, s l raheja hospital, raheja rugnalaya marg mahim, mumbai-400016, Tel: 00919004479549, E-mail: docsanjith@rediffmail.com

\begin{abstract}
Nutritional screening for malnutrition is the first step in the nutrition care plan process.There is multiple tools that have been utilized for nutritional screening. Many patients admitted to the intensive care units are known to have a very high risk for malnutrition. Various countries and also various intensive care units in different intensive care units in the same country also have different tools to screen out patients at risk for malnutrition. Among the most frequently used are the Nutric score, the SGA (subjective global assessment) and the NRS (nutrition risk screening) 2002.
\end{abstract}

We compared the level of agreement between the Nutric score, Nutritional Risk Screening (NRS) 2002 and Subjective Global Assessment (SGA) for nutritional risk assessment and for predicting length of ICU stay (LOS-ICU), length of hospital stay (LOS-HOSP) and in-hospital mortality.

\section{Introduction}

Patients admitted to the intensive care unit (ICU) are usually at high risk of malnutrition [1,2]. Critical illness is associated with a very high energy expenditure and an uncontrolled cachexia at times which leads to high amount of protein loss associated with weight loss. This resetting of the homeostasis leads to various other problems like electrolytes abnormality, increased length of hospital and ICU stay, increase duration on mechanical ventilator, reduction in muscle power, failure to thrive etc. These patents if not picked up early go onto a phase of prolonged acute critical illness followed by a phase of chronic critical illness. This causes marked reduction of quality of life. Hence identifying these patients early might help in tackling the issue by helping to reduce iatrogenic underfeeding or overfeeding. This demand for some form of screening tool to identify at-risk patients early. There are many tools, subjective and objective, but unfortunately there is no single standard tool used to assess nutritional status. The commonly used scores which have been validated include the Nutric score, the NRS 2002 and the SGA (subjective global assessment). The purpose of our study was to compare the accuracy of Nutric score, NRS 2002 and SGA in predicting LOSICU, LOS-HOSP and in-hospital mortality.

\section{Materials and Methods}

A total of 348 consecutive patients admitted between March to June 2016 in a mixed (medical/surgical) ICU were assessed on day of admission using the three screening tools separately to classify them into high-risk and low-risk of malnutrition. A trained intensive care doctor and a dietitian were involved in the data collection. Day 1 APACHE 2 scores and demographic data (weight, height, age, sex) were recorded. LOS-ICU (length of Stay in the ICU), LOS-HOSP (length of stay in the hospital) and inhospital mortality and secondary outcomes were studied, need for supplemental nutritional support (enteral/parenteral), need for ventilation (non-invasive/invasive) and need for dialysis in high-risk and low-risk patients by each nutrition assessment tool were studied. These data were all compiled using the "inutrimon" software which is a data management software developed by the author which helps in suggestion and optimization of feeding process All the data were compiled and analyzed with the Chi square test and t-test. Level of agreement (Kappa) and Odds ratio were also calculated.

\section{Results}

Of the 348 patients studied, 221 (63.5\%) were males and 127 $(36.5 \%)$ were females as seen in Table 1 . In males, $32.13 \%$ were at a low risk while $67.87 \%$ were at a high risk. In females, $28.35 \%$ were at a low risk while $71.65 \%$ were at a high risk. Chi square test was used and the $\mathrm{p}$ value was found to be 0.462 ( $\mathrm{p}>0.05)$.

Table 2 shows the chronic comorbidities and admission diagnosis of the patients. The mean APACHE 2 score for low risk patients was 8.04 with a standard deviation of 3.4 whereas for high risk patients it was 15.11 with a standard deviation of 6.1. Using the $T$ test the $p$ value was calculated to be $0.000(p<0.05)$ which is statistically significant. This suggests that patients with high risk of nutrition had a higher APACHE 2 score.

The Nutric score, NRS 2002 and SGA identified high-risk of malnutrition in $10.63 \%, 64.94 \%$ and $40.81 \%$ patients respectively 
as shown in Table 3. $67.87 \%$ males and $71.65 \%$ females were found to be at a high risk of malnutrition by at least one of the scores as shown in Table 1. The mean APACHE 2 score for patients at high risk (using any one screening tool) was 15.11 (SD 6.10) and 8.04 for the low risk group (SD 3.34; $\mathrm{p}<0.01$ ). 64.9 percent and 40.8 percent of patients were detected as high risk for malnutrition by NRS 2002 and SGA respectively, while only 10.6 percent patients were classified as high risk for malnutrition by the Nutric score. A statistically significant highest level of agreement (kappa score-0.38) was seen between SGA and NRS 2002 in screening out patients with high and low risk of malnutrition Table 4. The NRS 2002 and SGA demonstrated statistically significant correlation ( $p=0.001$ ) for length of ICU stay for both the high risk and low risk group whereas only the NRS 2002 correlated significantly for the length of hospital stay $(p=0.002)$. Mortality was significantly higher in high risk patients identified using all 3 scores but the odds ratio of mortality in high risk patients vs low risk patients was highest with the nutric was 168.7 as compared to 8.08 with NRS 2002 and 7.95 with SGA Table 5 .

Table 1: Baseline characteristics of 348 ICU patients at low risk and high risk of malnutrition

\begin{tabular}{|c|c|c|c|c|c|}
\hline \multicolumn{2}{|c|}{ Patient Characteristics } & \multirow{2}{*}{$\begin{array}{l}\text { Total } \\
221 \\
\end{array}$} & \multirow{2}{*}{$\begin{array}{l}\text { Low-risk group } \\
\qquad \mathbf{N}=\mathbf{1 0 7} \\
71(32.13 \%)\end{array}$} & \multirow{2}{*}{$\begin{array}{l}\text { High-risk group } \\
\qquad \mathbf{N}=\mathbf{2 4 1} \\
150(67.87 \%)\end{array}$} & \multirow{2}{*}{$\begin{array}{c}\text { P value } \\
0.462\end{array}$} \\
\hline \multirow{2}{*}{ Sex } & Males & & & & \\
\hline & Females & 127 & $36(28.35 \%)$ & $91(71.65 \%)$ & \\
\hline
\end{tabular}

Table 2: Chronic comorbidities and admission diagnosis

Chronic Comorbidities

\begin{tabular}{|c|c|c|}
\hline Diabetes Mellitus & $42(35.9 \%)$ & $97(30.4 \%)$ \\
\hline Hypertension & $41(35 \%)$ & $108(33.9 \%)$ \\
\hline Ischemic Heart Disease & $11(9.4 \%)$ & $37(11.6 \%)$ \\
\hline Chronic kidney disease & $4(3.4 \%)$ & $24(7.5 \%)$ \\
\hline CVA & $0(-)$ & $11(3.4 \%)$ \\
\hline PVD & $8(6.8 \%)$ & $7(2.2 \%)$ \\
\hline Carcinoma & $11(9.4 \%)$ & $35(11 \%)$ \\
\hline \multicolumn{3}{|l|}{ Admission diagnosis } \\
\hline Pneumonia & $0(-)$ & $17(26.2 \%)$ \\
\hline Stroke & $1(5.9 \%)$ & $2(3.1 \%)$ \\
\hline Post-operative status & $15(88.2 \%)$ & $29(44.6 \%)$ \\
\hline
\end{tabular}

Table 3: Number of patients classified as low-risk and high-risk by the 3 nutritional assessment score

\begin{tabular}{|c|c|c|}
\hline Nutrition assessment score & Low risk & High risk \\
\hline Nutric & $311(89.37 \%)$ & $37(10.63 \%)$ \\
\hline NRS 2002 & $122(35.06 \%)$ & $226(64.94 \%)$ \\
\hline Subjective global assessment (SGA) & $206(59.19 \%)$ & $142(40.81 \%)$ \\
\hline
\end{tabular}


Table 4: Agreement in classifying low-risks and high-risk patients between the 3 methods

\begin{tabular}{|c|c|c|c|c|c|c|c|}
\hline \multirow{3}{*}{ Assessment Method } & & \multicolumn{2}{|c|}{ NUTRIC } & \multicolumn{2}{|c|}{ NRS 2002} & \multicolumn{2}{|c|}{ SGA } \\
\hline & & Low-risk & High-risk & Low-risk & High-risk & Low-risk & High-risk \\
\hline & & $\mathrm{N}=311$ & $N=37$ & $\mathrm{~N}=122$ & $N=226$ & $N=206$ & $N=142$ \\
\hline \multirow{2}{*}{ NUTRIC } & $\begin{array}{l}\text { Low- } \\
\text { risk }\end{array}$ & & & 119 & 87 & 186 & 117 \\
\hline & $\begin{array}{l}\text { High- } \\
\text { risk }\end{array}$ & & & 1 & 36 & 14 & 22 \\
\hline \multirow{4}{*}{ NRS 2002} & \multirow{2}{*}{$\begin{array}{l}\text { Low- } \\
\text { risk }\end{array}$} & 122 & 0 & & & 107 & 15 \\
\hline & & $35.06 \%$ & $0.29 \%$ & & & $30.75 \%$ & $4.31 \%$ \\
\hline & \multirow{2}{*}{$\begin{array}{l}\text { High- } \\
\text { risk }\end{array}$} & 189 & 37 & & & 99 & 127 \\
\hline & & $54.31 \%$ & $10.63 \%$ & & & $28.45 \%$ & $36.49 \%$ \\
\hline \multirow{4}{*}{ SGA } & \multirow{2}{*}{$\begin{array}{l}\text { Low- } \\
\text { risk }\end{array}$} & 193 & 13 & 112 & 96 & & \\
\hline & & $55.46 \%$ & $3.73 \%$ & & & & \\
\hline & \multirow{2}{*}{$\begin{array}{l}\text { High- } \\
\text { risk }\end{array}$} & 118 & 24 & 12 & 126 & & \\
\hline & & $33.91 \%$ & $6.90 \%$ & & & & \\
\hline \multicolumn{8}{|c|}{ Nutric and NRS: Level of agreement (Kappa) is 0.121 . Although the value is low $(\mathrm{N}=0-1)$, it is statistically significant ( $\mathrm{p}=0.000)$} \\
\hline \multicolumn{8}{|c|}{ Nutric and SGA: Level of agreement (Kappa) is 0.120 . Although the value is low $(N=0-1)$, it is statistically significant $(\mathrm{p}=0.002)$} \\
\hline
\end{tabular}

Table 5: Outcomes in low-risk and high-risk patients

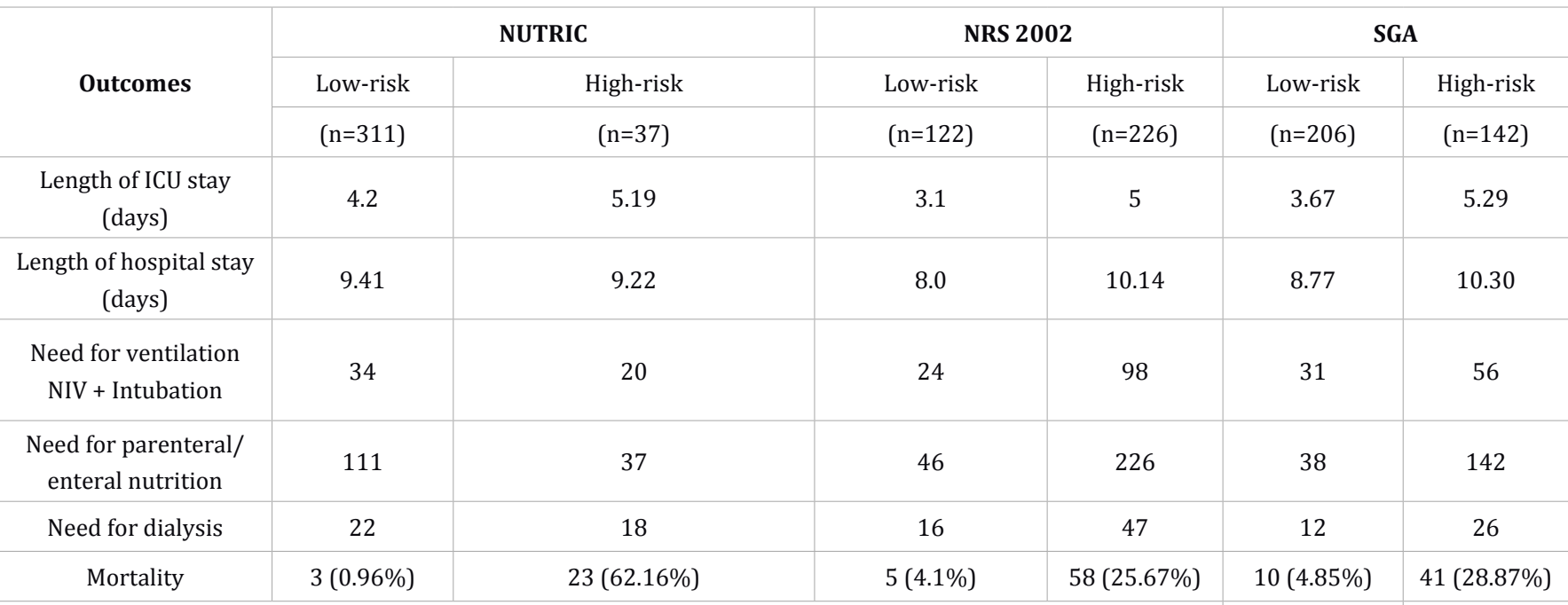

Note: $\mathrm{p}$ value versus low risk $=*<0.05 ; * *<0.005$

Nutric: Odds ratio was found to be 168.67 . This indicates odds of death among high risk group of patients were found to be around 168 times higher than the low risk group.

NRS 2002: Odds ratio was found to be 8.08. This indicates odds of death among high risk group of patients were found to be around 8 times higher than the low risk group.

SGA: Odds ratio was found to be 7.96. This indicates odds of death among high risk group of patients were found to be around 8 times higher than the low risk group. 


\section{Discusssion}

The Aspen and the SCCM have clearly explained the need to have nutritional screening tools to identify patients at high risk for malnutrition. They went on to recommend either the NUTRIC score or the NRS 2002 for this purpose [3]. Patients admitted to the ICU usually are likely to be at a high risk of malnutrition and this demands a tool to screen such patients early. Despite the availability of several subjective as well objective tools, there is no standardized measure to assess nutritional status. Hence the objective of this study was to compare the level of agreement between the Nutric Score, Nutritional Risk Screening (NRS), Subjective Global Assessment (SGA) in nutritional risk assessment and also to correlate the same to predicting primary patient outcomes in terms of Length of ICU Stay ( LOS - ICU), Length of Hospital Stay ( LOS - HOSP) and in hospital mortality as well as some secondary parameters such as need for supplemental nutritional support ( enteral/ parenteral) , need for ventilation ( invasive / non invasive) and need for dialysis.

The Nutric Score was the first nutritional risk assessment tool developed and validated specifically for ICU patients.The recognition that not all ICU patients will respond the same to nutritionalinterventions was the main concept behind the NUTRIC score, as most other risk scores and assessment tools consider all critically ill patients to be at high nutrition risk. NRS 2002 has been proposed on the basis of analysis of controlled clinical trials. It is designed to identify those who need nutritional support. This tool contains a severity of disease score, a nutritional score and an age score. Subjective Global Assessment, or SGA, is a proven nutritional assessment tool that has been found to be highly predictive of nutrition-associated complications. SGA fulfills the requirements of a desirable system of nutritional assessment by: Identifying malnutrition, Distinguishing malnutrition from a disease state, Predicting outcome, Identifying patients in whom nutritional therapy can alter outcomes. However in view of number of scores in place it is not clear as to which score should be used with various units all over the world using different scores as per their convenience. We attempted to understand whether or not there are any agreements between these various scores. In Gi surgery NRS 2002 score was closely related to the length of hospital stay, the incidence of complications, and the mortality [4].

Among the various tools the NRS 2002 seems to screen out more patients at risk for malnutrition and there seems to be some sort of level of agreement between the SGA and NRS 2002 in screening out those patient with high risk and low risk of malnutrition .They also seems to be a significant correlation between SGA and NRS 2002 when comparing to length of stay .The NUTRIC score seemed to predict mortality much better than the rest of the two scores.

\section{Conclusion}

There was a wide difference in the percent of patients identified as high-risk using each of the 3 scores. High risk when predicted by NRS 2002 and SGA also predicted increased LOS-ICU and LOC-HOSP, but mortality was best predicted by the NUTRIC score. However this study still does not answer he question as to which of the screening tools should be used in the ICU regularly to identify patients at risk for malnutrition. Moreover owing to the poor level of agreement between all the three scores it would be become very difficult to study epidemiology if various intensive care units use different screening tools. This study lays the impetus for devising a new screening tool that would be in close agreement to all the three frequently used screening tools studied. Use of such a new screening tools would help to standardize research and help us to target specialized nutritional support uniformly and thus study outcomes and other major relevant end points.

\section{References}

1. Correia MI, Campos AC, Study EC. Prevalence of hospital malnutrition in Latin America: The multicenter ELAN study. Nutrition. 2003;19(10):823-825.

2. Waitzberg DL, Caiaffa WT, Correia MI. Hospital malnutrition: the Brazilian national survey (IBRANUTRI): a study of 4000 patients. Nutrition. 2001;17(7-8):573-580.

3. McClave SA, Taylor BE, Martindale RG, et al. Guidelines for the provision and assessment of nutrition support therapy in the adult critically ill patient: Society of Critical Care Medicine (SCCM) and American Society for Parenteral and Enteral Nutrition (A.S.P.E.N.). JPEN J Parenter Enteral Nutr. 2016;40( 2):159 -211. Doi: 10.1177/0148607115621863

4. Sorensen J, Kondrup J, Prokopowicz J, Schiesser M, Krähenbühl L, Meier R, et al. EuroOOPS: An international, multicentre study to implement nutritional risk screening and evaluate clinical outcome. Clin Nutr. 2008;27(3):340-349. Doi: 10.1016/j.clnu.2008.03.012 